\title{
Projeto Memória e Envelhecimento: capacitando profissionais e aprimorando aspectos cognitivos em idosos institucionalizados
}

\author{
Edvaldo Soares*, Tamara Pereira de Oliveira"*, Patrícia Sayuri Komatsu**, Stella Maira Demartini"**, \\ Michele Mita Suziki"*, Mirela Mendes Rodrigues"
}

\section{Resumo}

O aumento da população idosa é um fenômeno mundial e traz consequências diversas, não só em termos de saúde, mas também em termos econômicos. Uma dessas consequências é a institucionalização. O projeto, em execução em duas instituições residenciais de longa permanência para idosos (IRLPi) localizadas na cidade de Marília - SP, tem como objetivos: a) capacitar equipe multidisciplinar para atuar em IRLPi's; b) elaborar e promover atividades que melhorem a qualidade de vida, com enfoque especial na preservação da saúde mental; c) estudar as condições de saúde geral e mental dos idosos institucionalizados. O projeto foi implantado em três fases: 1 - coleta de dados e elaboração de plano de atividades; 2 - aplicação de atividades; 3 - avaliação/análise de resultados. Em relação à fase 1 , os dados foram colhidos por meio de três formulários, mediante os quais se verificaram dados institucionais, dados sociais e saúde geral. Os dados referentes à saúde foram colhidos a partir da medicação de uso contínuo. Todos os dados foram lançados em banco de dados (Access) e submetidos à análise estatística descritiva. Dados referentes à saúde mental foram confirmados pela aplicação de testes clínicos da escala geriátrica de depressão e do Mini-Exame do Estado Mental (GDE e MMSE-T nas siglas em inglês).

Palavras-chave: Envelhecimento. Institucionalização. Cognição. Cuidadores.

* Doutor em Neurociências e Comportamento pela Universidade de São Paulo. Professor do Departamento de Psicologia da Educação da Universidade Estadual Paulista Júlio de Mesquita Filho: Campus de Marília - SP. Líder do Grupo de Estudos em Neurociência Cognitiva e Envelhecimento Humano. Coordenador do Projeto de Extensão Memória e Envelhecimento. Endereço para correspondência: Centro de Pesquisa e Ensino em Neurociências, Universidade do Estado de São Paulo: Campus de Marília - SP, Av. Vicente Ferreira, 1278. CEP: 17515-000, Marília - SP. E-mail: edsoares@marilia.unesp.br.

** Graduadas em Terapia Ocupacional pela Faculdade de Filosofia e Ciências da Universidade Estadual Paulista Júlio de Mesquita Filho: Campus de Marília - SP. Membros do Grupo de Estudos em Neurociência Cognitiva e Envelhecimento Humano. Participantes do Projeto de Extensão Memória e Envelhecimento. Bolsistas Proex.

*** Acadêmicas do curso de Terapia Ocupacional da Faculdade de Filosofia e Ciências da Universidade Estadual Paulista Júlio de Mesquita Filho: Campus de Marília - SP. Membros do Grupo de Estudos em Neurociência Cognitiva e Envelhecimento Humano. Participantes do Projeto de Extensão Memória e Envelhecimento.

$\longrightarrow$ Recebido em março de 2009 - Avaliado em novembro de 2009.

$\rightarrow$ doi:10.5335/rbceh.2010.007 


\section{Introdução}

O crescimento da população idosa nos países desenvolvidos vem sendo estudado há vários anos, ao passo que nos países do Terceiro Mundo só recentemente o tema tem despertado interesse. (VERAS et al., 1988). No Brasil, os idosos, que representavam apenas $3,2 \%$ da população geral de 1900 e 4,7\% em 1960, poderão atingir $13,8 \%$ em 2025 . Hoje, temos aproximadamente 11 milhões de pessoas com mais de sessenta anos (idosos) e projeções indicam que seremos o sexto país do mundo em número de idosos em 2020, com aproximadamente 32 milhões de idosos. Esse crescimento decorre, segundo Silva (2003), da industrialização, da urbanização, dos avanços da medicina, da tecnologia e do saneamento básico. Tais fatores se configuram como condições que reduzem a fecundidade e a mortalidade e favorecem o aumento da expectativa de vida, resultando, assim, no crescimento da população idosa e, consequentemente, no envelhecimento demográfico.

Com o envelhecimento demográfico, temos um aumento dos gastos previdenciários, uma maior demanda pelos serviços de saúde e, consequentemente, aumento nos custos destes serviços. Ainda em decorrência desse crescimento, não raramente, nos deparamos com problemas de ordem social, financeira e com dúvidas sobre o que fazer com os idosos, que frequentemente apresentam diversas patologias características da idade. Tais problemas afetam não só o Estado e o próprio idoso, mas também as famílias.
Em razão das mudanças dos parâmetros sociais e econômicos, hoje muitas famílias não conseguem manter o idoso no ambiente familiar, pois, além de não terem uma estrutura adequada para suprir as necessidades do idoso, estão impossibilitadas de conciliar as atividades laborais e pessoais com os cuidados demandados por este. Muitas vezes se soma a tal quadro o desejo do idoso de encontrar um local que lhe proporcione atenção, conforto e, especialmente, atendimento às suas necessidades básicas.

Em tais condições, o idoso é muitas vezes visto como um entrave à autonomia familiar, o que torna precária a relação entre ele e seus familiares. Muitas vezes a solução encontrada, tanto pela família como pelo idoso, é a institucionalização ${ }^{1}$. (PERLINI; LEITE; FURINI, 2007). Dessa forma, mesmo sendo ainda a maioria da população idosa independente e residindo na comunidade, uma minoria precisa contar com o apoio de instituições residenciais de longa permanência para idosos (IRLPis), onde muitas vezes as atividades mentais não são devidamente estimuladas. Em tais circunstâncias, o idoso, quase sempre, tende a acumular problemas sociais e de saúde.

Foi nesse contexto que surgiu o interesse em desenvolver pesquisas que tivessem como eixo central o idoso institucionalizado. Porém, juntamente com interesse pela pesquisa, surgiram alguns questionamentos de ordem ética: Qual a contribuição efetiva que tal pesquisa trará a tal população? Seria ético somente "usar" os idosos como sujeitos de pesquisa e nada oferecer a curto prazo?

Desses questionamentos surgiu a ideia de um projeto de extensão que 
atendesse diretamente a população idosa e, então sim, desenvolver atividades de pesquisa. Mas como desenvolver tal projeto envolvendo pesquisa e extensão? Para solucionar tal problema de ordem prática, resgatamos o método baseado em três ações: ver, julgar e agir, ou seja, procuramos, em primeiro lugar, conhecer o ambiente e os sujeitos; em segundo, avaliar a situação dos sujeitos e suas necessidades e, por fim, elaborar, dentro dos interesses e competências do grupo, um plano de ação que atendesse às demandas das instituições e dos sujeitos envolvidos. (SOARES, 2003). Assim, teve início o projeto "Memória e Envelhecimento: aspectos neuropsicológicos e estratégias preventivas".

O projeto, em funcionamento desde 2006, objetiva, além de capacitar cuidadores, elaborar e aplicar atividades preventivas às patologias de caráter demencial, visando principalmente minimizar fatores de risco associados e, assim, melhorar a qualidade de vida dos internos. Dessa forma, o enfoque da proposta centra-se em atividades relacionadas às funções cognitivas.

O projeto foi implantado em três fases. Na primeira, além dos contatos preliminares e da seleção dos alunos voluntários, foram colhidos dados relativos às duas IRLPis, tais como: esquema de visitas; relação espaço físico-número de internos (alojamento, internos por quarto, etc.); infraestrutura (adaptação do espaço físico às limitações do idoso, equipamentos, área de lazer, etc.); atividades diversas (físicas, culturais, lazer, etc.); padrão socioeconômico dos usuários (internos); motivo da internação; meios para coleta de dados em relação à saúde geral dos internos; levantamento dos projetos de extensão e estágios em funcionamento na instituição. Os dados foram lançados em formulários elaborados pelos integrantes do projeto ${ }^{2}$.

$\mathrm{Na}$ segunda fase os dados obtidos na fase anterior foram analisados e discutidos. Também foi elaborado um Plano de Atividades e Cronograma de trabalhos nas IRLPs, bem como definida a(s) equipe(s) de trabalho. Na terceira fase, com base nos levantamentos feitos acerca do interesse, estrutura e necessidade da instituição, iniciaram-se as atividades práticas e de pesquisa. Nesta fase, além das sessões com os idosos, foram colhidos dados acerca da saúde geral e mental, bem como se elaboraram prontuários dos internos, os quais são atualizados bimestralmente ${ }^{3}$.

\section{A institucionalização e as instituições objeto do projeto}

Para muitos autores os asilos geralmente são vistos como um "caminho sem volta”, considerando que tanto a família como a comunidade se esquecem dos idosos internados. Esses idosos, por sua vez, acabam perdendo a identidade e a cidadania, em conseqüência das condutas e comportamentos regidos pelas normas das instituições. (SCHMITZ, 1997 apud GUIMARÃES; SIMAS; FARIAS, 2005; ZIMERMAN, 2000). Segundo Guimarães, Simas e Farias (2005), as IRLPis apresentam aspectos próprios e determinam condutas e comportamentos pelas normas vigentes e institucionais. $\mathrm{Na}$ maioria dessas instituições as atividades são realizadas num mesmo ambiente e sob uma única autoridade; a rotina é praticamente igual para todos, sendo 
ignoradas diferenças individuais, bem como a história da vida de cada um.

As IRLPis comprometem-se em suprir as necessidades básicas de moradia, alimentação, saúde e convivência social, ou seja, uma melhor qualidade de vida. Todavia, nem sempre esse compromisso é cumprido. "A institucionalização para a grande maioria dos idosos é fonte de dor e tristeza, o ambiente se torna silencioso, indiferente, vazio, passando a representar os momentos finais de sua vida podendo contribuir para o maior problema psicológico do idoso: a depressão." (PARMELEE; KATZ; LAWTON, 1989, apud MARTINS, 2005, p. 69).

Zimerman (2000) observa que, além da dificuldade que o idoso tem para se acostumar à nova realidade, outros problemas podem ocorrer, tais como mudança interna de parâmetros, convivência, perdas, falta de convivência com o sexo oposto, abandono, entre outros.

$\mathrm{O}$ idoso institucionalizado não raras vezes apresenta uma série de comportamentos negativos, tais como diminuição da capacidade funcional, perda da função, dificuldade na realização das atividades de vida diária, baixa interação social, motivação reduzida. Os cuidados prestados em tais instituições podem muitas vezes ser inadequados, ineficientes e mesmo inexistentes, quando os cuidadores são despreparados ou sobrecarregados. (CALDAS apud CALDAS, 2003).

Apesar de muitos autores considerarem as instituições asilares como locais inapropriados, deve-se considerar que, na inexistência desses locais, muitos idosos estariam morando na rua ou sendo mal atendidos pelos familiares.
As duas IRLPis nas quais o projeto é desenvolvido atende especialmente esses idosos mais desvalidos. A Instituição 1, fundada em 1982, tem como filosofia de trabalho a assistência aos idosos, sem distinção de qualquer natureza. A captação de recursos, como ocorre na maioria das IRLPis, se dá principalmente por meio de doações. Situa-se em um bairro da periferia e tem cerca de 800 $\mathrm{m}^{2}$ de área construída. Os idosos são distribuídos em 18 apartamentos, todos com banheiro. Em cada apartamento habitam, em média, dois idosos; mas há casos em que idosos ocupam o espaço individualmente. Há um refeitório e uma cozinha, bem como um ambulatório. Existe espaço destinado à fisioterapia e uma lavanderia. Adaptações foram realizadas (instalação de corrimões, ventilação, campainhas, rampas, etc.).

Em relação aos recursos humanos para o atendimento aos idosos, há na Instituição 1 uma enfermeira, cinco auxiliares de enfermagem, uma cozinheira e 11 auxiliares de serviços gerais. Em termos de programas de atendimento e prevenção realizados na instituição, havia até o segundo semestre de 2008 apenas a realização de estágio dos alunos do curso de Fisioterapia da Faculdade de Filosofia e Ciências (FFC) da Universidade Estadual Paulista (Unesp) - Campus de Marília. A partir do segundo semestre de 2008, iniciaram-se atividades de estágio em terapia ocupacional. Segundo relato dos próprios administradores, até o ano de 2006 havia carência desse tipo de atividades na instituição. No ano de 2007 eram atendidos 47 idosos na Instituição 1. Em termos de gênero, há uma pequena predominância de idosos do 
sexo masculino (57\%). A média de idade dos idosos é de 71,0 anos de idade, com as mulheres tendo média de 68,8 anos e os homens, 72,9 anos (Figura 1).

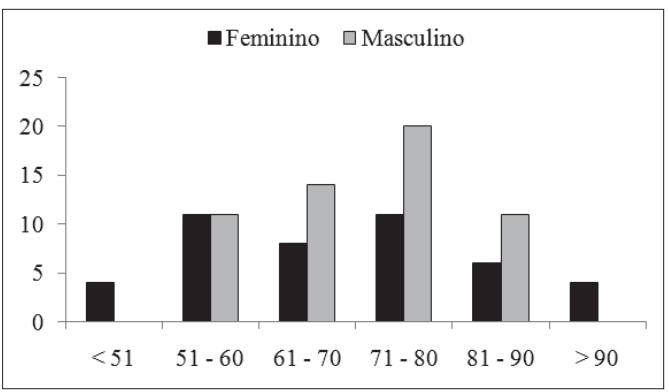

Figura 1 - Distribuição dos idosos na Instituição 1 por faixa etária.

A Instituição 2, fundada em 1930 e localizada em região mais central da cidade, tem como filosofia de trabalho atendimento ao idoso acima de 65 anos de origem carente. A captação de recursos ocorre por meio de doações, convênios com os governos estadual e federal, aluguéis, campanhas, festas beneficentes, etc. O índice de ocupação dos quartos é de dois ou três idosos por quarto. Ao contrário da Instituição 1 , há divisão entre os sexos, ou seja, os idosos do sexo masculino ocupam uma ala e os do sexo feminino, outra. Na ala feminina existem 17 leitos, 16 banheiros, uma copa e um refeitório; na ala masculina, são 24 leitos, 18 banheiros e uma sala de televisão. Há uma lavanderia com três salas, dois despejos de roupas e um banheiro. Existe uma área reservada à fisioterapia, que comporta quatro salas de atendimento, um banheiro, e uma sala de espera. Adaptações foram realizadas (instalação de corrimões, campainhas, rampas, etc.). Também há uma ala reservada para atividades de terapia ocupacional e fonoaudiologia.

Em relação aos recursos humanos para o atendimento aos idosos, existem um médico, uma cozinheira, 18 auxiliares de serviços gerais, um pedreiro, uma secretária, um auxiliar de secretária, um porteiro, um servente e um assistente social. Em termos de programas de atendimento e prevenção realizados na instituição, há somente a realização de estágio dos alunos do curso de Terapia Ocupacional da FFC/ Unesp - Campus de Marília, e dos cursos de Fisioterapia e Psicologia de uma outra instituição de ensino superior da cidade de Marília. São atendidos 69 idosos. Em termos de gênero, ao contrário da Instituição 1 , há predominância de indivíduos do sexo feminino (55\%). A Figura 2 apresenta a distribuição dos idosos da Instituição 2 em termos de faixa etária.

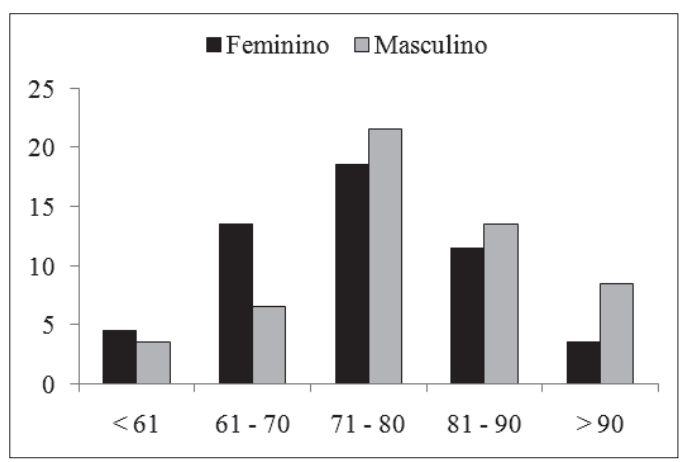

Figura 2 - Distribuição dos idosos na Instituição 2 por faixa etária.

Em termos gerais, dos 116 idosos pesquisados, quanto ao gênero, foram exatamente $50 \%$ homens e $50 \%$ mulheres. Desses 116 idosos, 56,2\% têm mais de setenta anos de idade. 


\section{Os sujeitos: aspectos demográficos ${ }^{4}$}

O levantamento das características gerais dos idosos teve por objetivo conhecer a realidade desses, pois sem tal conhecimento seria improvável elaborar um plano de atividades que atendesse às expectativas das IRLPis envolvidas como dos próprios idosos. Em relação ao tempo de institucionalização, considerados os 116 idosos internos nas instituições 1 e 2 , observamos que há uma variação (Figura 3). Porém, a distribuição mostra que 46,4\% deles estão internados há menos de cinco anos.

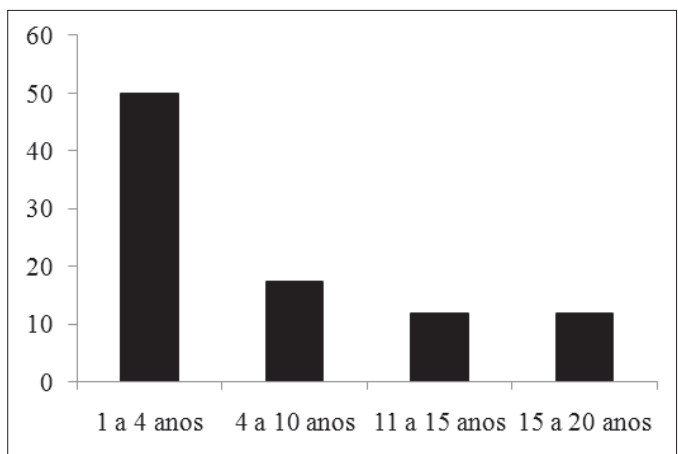

Figura 3 - Tempo médio de institucionalização.

A pesquisa demonstrou também haver baixo índice de rotatividade entre os idosos. Os motivos de desligamento referem-se, principalmente, à ocorrência de óbitos, internações, sobretudo em instituições para tratamento psiquiátrico, e raramente para retornarem ao seio familiar (Figura 4).

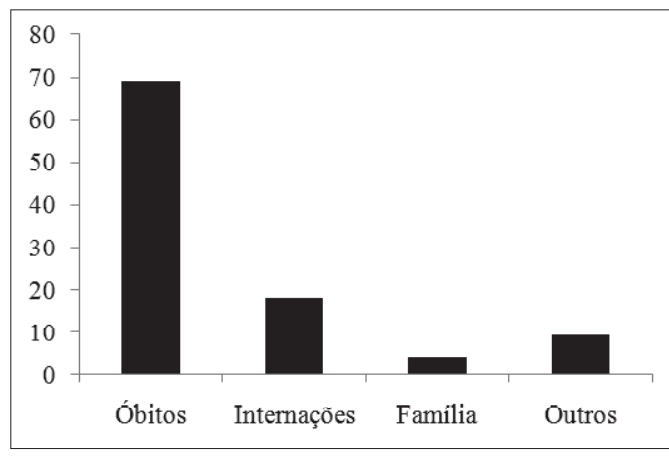

Figura 4 - Motivos de desligamento.

A origem dos idosos pesquisados, em termos regionais, é variável, mas a maioria é oriunda da região Sudeste $(61,6 \%)$. Porém, há significativo número de idosos oriundos da região nordeste do Brasil (Figura 5).

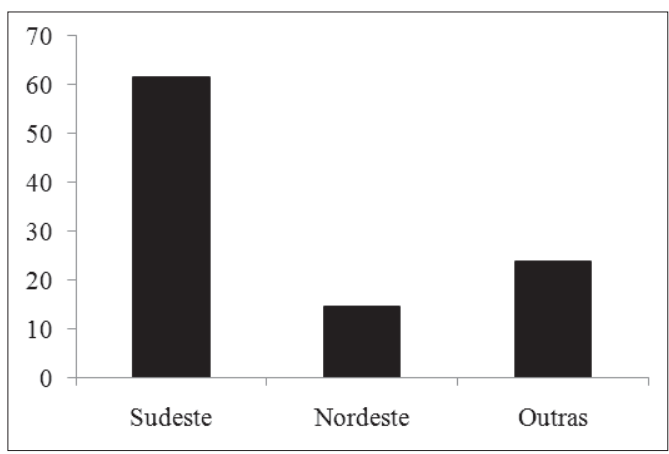

Figura 5 - Região de nascimento.

Em relação ao estado civil, prevalecem os idosos que se declararam solteiros $(56 \%)$ ou viúvos (44\%). Não houve declarações acerca de separações/divórcios e não há registros nas instituições, apesar de haver relato dos cuidadores. Por isso, consideramos apenas o relato dos próprios idosos, mesmo sabendo que tais números são inexatos. Do total, somente $21,5 \%$ declaram ter filho(s). No 
que se refere ao recebimento de visitas (parentes e amigos), um dado nos chamou a atenção: $60,2 \%$ do total dos idosos não recebem qualquer tipo de visita. No tocante à escolaridade, há a predominância de analfabetos $(72 \%)$ e, em relação à etnia, há predominância de indivíduos brancos (Figura 6).

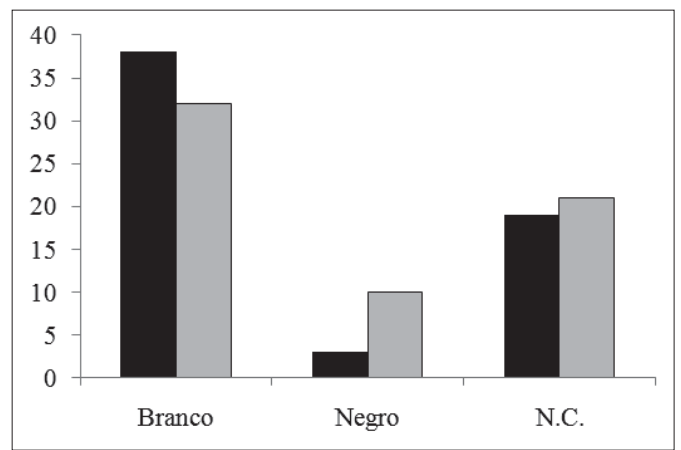

Figura 6 - Distribuição dos idosos por etnia.

\section{Os sujeitos: a saúde}

O envelhecimento deve ser entendido como um processo natural de desgaste fisiológico dos sistemas do organismo. Tal desgaste pode ocasionar diminuição das capacidades físicas, aumento relativo da incapacidade para realização de atividades cotidianas, modificações morfológicas, bioquímicas, psicológicas, perda de papéis sociais, solidão, perdas afetivas e aumento da vulnerabilidade, diminuição da capacidade de recuperação dos efeitos de eventos que desequilibram o organismo (resiliência), menor plasticidade neural/comportamental. (COHEN, 1995; STUART-HAMILTON, 2002; SOARES, 2006).

O ritmo desse processo e o impacto sobre a qualidade de vida dependem de fatores econômicos, genéticos, demográficos, comportamentais e socioculturais. A institucionalização pode ser um fator de impacto negativo na saúde do idoso e, aliada a ela, o consumo excessivo de medicamentos. Em termos de saúde geral dos idosos pesquisados ${ }^{5}$ nas duas IRLPIs, foi possível destacar dois fatores de grande impacto no declínio da qualidade de vida do idoso: a polipatologia e a polimedicação, conforme se pode observar nas tabelas 1, 2 e 3 .

Tabela 1 - Quadro geral de saúde com relação às patologias e queixas mais comuns.

\begin{tabular}{lrrrrr}
\hline \multirow{2}{*}{\multicolumn{1}{c}{ Patologias e queixas mais comuns }} & \multicolumn{2}{c}{ Feminino } & \multicolumn{2}{c}{ Masculino } & \multirow{2}{*}{ Total } \\
\cline { 2 - 5 } & \multicolumn{1}{c}{$\mathrm{n}$} & \multicolumn{1}{c}{$\%$} & $\mathrm{n}$ & \multicolumn{1}{c}{$\%$} & \\
\hline Diabetes & 8 & 80,0 & 2 & 20,0 & 10 \\
Hipertensão & 41 & 55,4 & 33 & 44,6 & 74 \\
Patologias cardíacas & 10 & 45,5 & 12 & 54,5 & 22 \\
Tireóide & 8 & 88,9 & 1 & 11,1 & 9 \\
Acidente vascular encefálico & 3 & 30,0 & 7 & 70,0 & 10 \\
Úlcera gástrica & 12 & 50,0 & 12 & 50,0 & 24 \\
Neoplasias & 1 & 7,1 & 13 & 92,9 & 14 \\
Problemas respiratórios & 2 & 20,0 & 8 & 80,0 & 10 \\
Contusões, traumas, reumatismo e osteoporose & 10 & 71,4 & 4 & 28,6 & 14 \\
Disfunções renais & 1 & 33,3 & 2 & 66,7 & 3 \\
Anemia & 2 & 100,0 & 0 & 0,0 & 2 \\
Dor crônica & 12 & 85,7 & 2 & 14,3 & 14 \\
\hline
\end{tabular}


Tabela 2 - Quantidade de medicamentos de uso contínuo administrados por dia.

\begin{tabular}{crrrrr}
\hline \multirow{2}{*}{ Medicamentos } & \multicolumn{2}{c}{ Feminino } & \multicolumn{2}{c}{ Masculino } & \multirow{2}{*}{ Total } \\
\cline { 2 - 5 } & \multicolumn{1}{c}{$\mathrm{n}$} & $\%$ & $\mathrm{n}$ & $\%$ & \\
\hline$\leq 1$ & 7 & 36,8 & 12 & 1,9 & 19 \\
2 a 3 & 23 & 53,5 & 20 & 1,2 & 43 \\
4 a 5 & 16 & 50,0 & 16 & 1,6 & 32 \\
6 a 7 & 8 & 53,3 & 7 & 3,6 & 15 \\
$\geq 8$ & 3 & 50,0 & 3 & 8,3 & 6 \\
\hline
\end{tabular}

Tabela 3 - Tipo de medicação de uso contínuo administrada.

\begin{tabular}{lrrrrr}
\hline \multirow{2}{*}{\multicolumn{1}{c}{ Variáveis }} & \multicolumn{2}{c}{ Feminino } & \multicolumn{2}{c}{ Masculino } & \multirow{2}{*}{ Total } \\
\cline { 2 - 5 } & \multicolumn{1}{c}{$\mathrm{n}$} & \multicolumn{1}{c}{$\%$} & \multicolumn{1}{c}{$\mathrm{n}$} & $\%$ & \\
\hline Tomam medicamentos & 54 & 51,9 & 50 & 48,1 & 104 \\
Não tomam medicamentos & 3 & 27,3 & 8 & 72,7 & 11 \\
Tomam medicação de uso psiquiátrico & 31 & 43,1 & 41 & 56,9 & 72 \\
Não tomam medicação de uso psiquiátrico & 13 & 40,6 & 19 & 59,4 & 32 \\
Só tomam medicação psiquiátrica & 2 & 28,6 & 5 & 71,4 & 7 \\
Só tomam medicação de uso geral & 20 & 62,5 & 12 & 37,5 & 32 \\
Tomam medicação de uso geral e psiquiátrico & 29 & 44,6 & 36 & 55,4 & 65 \\
\hline
\end{tabular}

Antes de apresentar os dados relativos à saúde mental dos idosos pesquisados, é importante salientar ainda que, de forma geral, o declínio da capacidade cognitiva (DCC) também decorre dos processos fisiológicos naturais. $\mathrm{Na}$ população idosa em geral, o que mais observamos é um comprometimento cognitivo leve. A identificação de indivíduos com potencial de risco torna-se fundamental, tanto em termos de prevenção como de posterior terapia. (CHARCHAT-FICHMAN et al., 2005). Foi nesse sentido que se realizamos nas IRLPIs pesquisadas levantamento da saúde mental dos idosos atendidos, mediante o qual observamos que um número significativo de idosos apresentava alguma alteração em termos de saúde mental, conforme podemos observar na Tabela 4 .

Tabela 4 - Saúde mental.

\begin{tabular}{lrrrrr}
\hline \multirow{2}{*}{\multicolumn{1}{c}{ Variáveis }} & \multicolumn{2}{c}{ Feminino } & \multicolumn{2}{c}{ Masculino } & \multirow{2}{*}{ Total } \\
\cline { 2 - 5 } & $\mathrm{n}$ & \multicolumn{1}{c}{$\%$} & $\mathrm{n}$ & $\%$ & \\
\hline Depressão & 25 & 58,1 & 18 & 41,9 & 43 \\
Ansiedade e psicoses & 24 & 64,9 & 13 & 35,1 & 37 \\
Quadros demenciais & 8 & 61,5 & 5 & 38,5 & 13 \\
Parkinson & 7 & 70,0 & 3 & 30,0 & 10 \\
Queixas de falhas em relação à memória & 20 & 69,0 & 9 & 31,0 & 29 \\
\hline
\end{tabular}


Em termos de manutenção da saúde, acreditamos que o estímulo para um bom funcionamento mental, físico e social configura-se como princípio para a promoção de saúde de idosos e, consequentemente, de prevenção não só das demências, graves ou leves, mas da saúde em seu conjunto. É com base nesse princípio que as atividades com os idosos estão sendo desenvolvidas.

\section{Ação: o projeto}

Com base nas informações colhidas nas fases iniciais, pudemos identificar com mais clareza quais são as principais demandas, as expectativas, tanto por parte das IRLPIs como dos próprios idosos, e, com base nessa identificação, discutir e elaborar atividades com os idosos, como, por exemplo, a realização de jogos (Figura 7.)

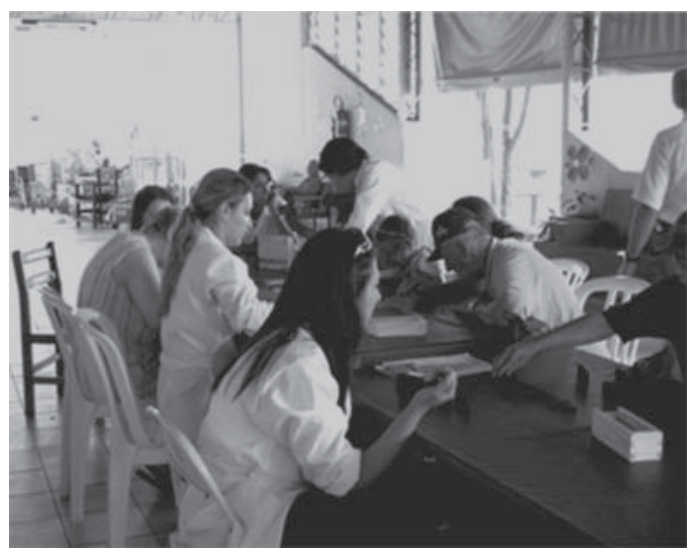

Figura 7 - Atividades práticas - jogos (2007).

Essas atividades deveriam levar em consideração alguns fundamentos: a) não "infatilização" do idoso; b) respeito às suas vontades; c) ver no idoso uma pessoa, com uma história, com anseios, desejos, limitações e potencialidades, não como um "paciente" e/ou como um "objeto de pesquisa"; d) compromisso; e) ética com relação às informações. Com base nesses fundamentos e nas reais necessidades identificadas, as atividades práticas foram desenvolvidas levando em consideração os seguintes princípios: a) atividades que promovessem a interação social entre os idosos de ambos os sexos; b) atividades que estimulassem cognitivamente; c) atividades que promovessem a integração entre os idosos e os participantes do projeto. Das atividades propostas destaca-se a atividade de dobradura (Figura 8).

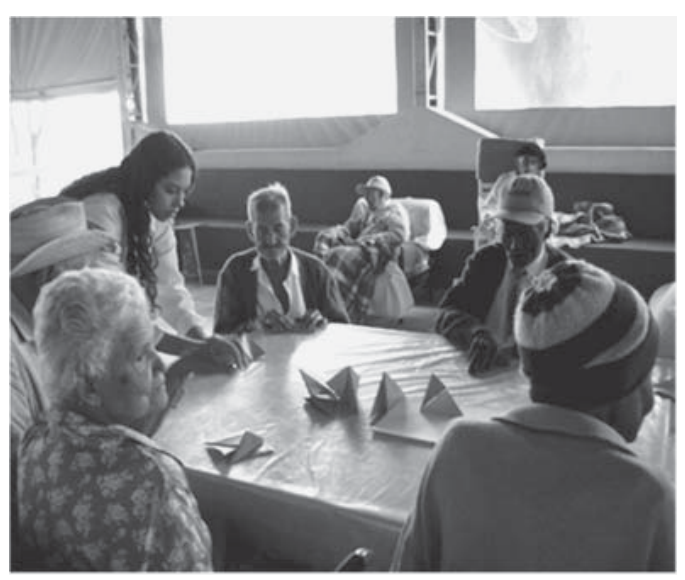

Figura 8 - Atividades práticas - dobradura (2008).

Nesses mais de dois anos de funcionamento foram realizadas 112 sessões de atividades (49 na Instituição 1 e 63 na Instituição 2). Cada sessão tinha duração média de quatro horas, totalizando em torno de 448 horas de atividades, as quais envolveram: aplicação de atividades práticas, entre as quais desenhos livres e direcionados, jogos, 
filmes, pintura e, principalmente, muita conversa; e de pesquisa (aplicação de testes, entrevistas). Também foi realizado um trabalho específico com cuidadores, o qual se constituiu em apresentação de resultados de pesquisa e atividades formativas visando a uma melhor capacitação para o cuidado. Participaram das atividades práticas, direcionadas à memória, cerca de cinquenta idosos do total de internos. ${ }^{6}$ Passaram pelo projeto como voluntários 34 alunos. Só no ano de 2008 participaram vinte alunos dos cursos de Fisioterapia (oito alunos), Terapia Ocupacional (oito alunos) e Psicologia ${ }^{7}$ (quatro alunos).

\section{Considerações finais}

Os dados coletados oferecem uma amostra da situação geral do idoso institucionalizado, confirmando em grande parte os dados levantados na literatura especializada, especialmente no que tange à saúde geral e mental. Porém, são frios. Devemos considerar que tais dados se referem a pessoas; seres humanos com as quais procuramos conviver. O início dessa convivência não foi fácil. Passamos ambos, executores, instituições e idosos, por uma fase de adaptação de conhecimento que, de certa forma, ainda perdura. Como pudemos notar, grande parte dos idosos apresenta, entre outras, limitações em termos de saúde que dificultam, ou mesmo impedem, sua participação nas atividades e, ainda, de manter um contato com os executores.

A pergunta essencial a ser respondida nesse artigo seria: O projeto tem melhorado a qualidade de vida dos idosos?
A nossa resposta é "ainda não sabemos", apesar de notarmos algumas mudanças significativas no comportamento de alguns idosos que participaram das atividades. Porém, tais mudanças podem ter ocorrido em função de outras variáveis. A resposta a tal pergunta depende de mais pesquisas, com características transversais e longitudinais, o que nem sempre é possível, dadas as características da população e o número de variáveis a serem consideradas.

Entretanto, uma coisa nós sabemos: a execução do projeto está sendo benéfica para nós pesquisadores. Houve uma mudança de comportamento, de visão acerca do envelhecimento por parte de nós, executores do projeto. Muitos preconceitos foram deixados de lado e muito temos aprendido com os idosos, a partir de uma relação de amizade e respeito. É nesse sentido que acreditamos que estamos no caminho certo e que o princípio de que "o estímulo para um bom funcionamento mental, físico e social configura-se como princípio para a promoção de saúde de idosos e, consequentemente, de prevenção às demências, graves ou leves" é um princípio válido. Porém, a elaboração de estratégias preventivas deve estar acompanhada do sentido de humanização. 
Project Memory and Aging: training professionals and improving cognitive aspects in patients under elderly care institution programs

\section{Abstract}

The increase in elderly population is a worldwide phenomenon and has different consequences not only in terms of health, but also in economic terms. One of those consequences is the institutionalization. The project, running in two elderly care institutions (hereafter IRLPi) located in Marília - SP aims to: a) Train multidisciplinary team to work in IRLPi's; b) Develop and promote activities that improve life quality focusing on the preservation of mental health; c) study general and mental health conditions of patients under elderly care institution programs. The project was implemented in three phases: a) data collection and elaboration of plan of activities; b) Application of activities; and c) assessment/ analysis of results. Regarding Phase 1, data were collected from 3 forms, through which it was possible to verify: Institutional Data; Social Data and General Health. The data related to health were collected from the medication of continuous use. All data were launched into the database (Access) and subjected to the descriptive statistical analysis. Data related to mental health were confirmed from the application of clinical trials (Geriatric Depression Scale EGD, and MMSE-T).

Key words: Aging. Institutionalization. Cognition. Caregivers.

\section{Referências}

CALDAS, C. P. Envelhecimento com dependência: responsabilidades e demandas da família. Caderno de Saúde Pública, Rio de Janeiro, v. 19, n. 3, p. 773-781, maio/jun. 2003.

CHARCHAT-FICHMAN, H. et al. Decline of cognitive capacity during aging. Revista Brasileira Psiquiatria, São Paulo, v. 27, n. 1, p.79-82, Mar. 2005.

COHEN, G. D. O cérebro no envelhecimento humano. São Paulo: Andrei, 1995.

GUIMARÃES, A. C. A.; SIMAS, J. P. N.; FARIAS, S. F. O ambiente asilar e a qualidade de vida nos idosos. A Terceira Idade, São Paulo, v. 16, n. 33, p. 54-71, jun. 2005.

PAMERLEE, P. A.; KATZ, I. R.; LAWTON, M. P. Depression among institutionalized aged: assessment and prevalence estimation. Journal of Gerontology, v. 44, s. n, p. 22-29, 1989.

PAVARINI, S. C. I. Dependência comportamental na velhice: uma análise do cuidado prestado ao idoso institucionalizado. 1996. [s. f.]. Tese (Doutorado em Educação) - Faculdade de Educação da Universidade Estadual de Campinas. Universidade Estadual de Campinas, Campinas: 1996.

PERLINI, N. M. O. G.; LEITE, M. T.; FURINI, A. C. Em busca de uma instituição para a pessoa idosa morar: motivos apontados por familiares. Revista da Escola de Enfermagem da Universidade de São Paulo, São Paulo, v. 41, n. 2, p. 229-236, jun. 2007.

SILVA, J. C. Velhos ou idosos? A Terceira Idade, São Paulo, v. 14, n. 26, p. 94-111, jan. 2003.

SOARES, E. Memória e envelhecimento: aspectos neuropsicológicos e estratégias preventivas. 2006. Disponível em: <www. psicologia.com.pt/artigos/textos/A0302.pdf>. Acesso em: 5 fev. 2009.

SOARES, E. Metodologia científica: lógica, epistemologia e normas. São Paulo: Ática, 2003. 
STUART-HAMILTON, I. A psicologia do envelhecimento: uma introdução. Porto Alegre: Artmed, 2002.

VERAS, R. P. et al. Research into elderly populations: the importance of the instrument and the training of the team: a methodological contribution. Revista de Saúde Pública, São Paulo, v. 22, n. 6, p. 513-518, Dec. 1988.

ZIMERMAN, G. I. Velhice: aspectos biopsicossociais. Porto Alegre: Artmed, 2000.

\section{Notas}

1 De acordo com Pavarini (1996), quando a família decide pela institucionalização do idoso, em geral, baseia-se em um ou mais dos três significados dados aos asilos: asilo-isolamento (resulta da concepção dada pelo senso comum como "depósito de velhos abandonados”); asilo-saúde (principalmente para idosos de baixa renda, que o associam com o hospital, onde possivelmente teriam melhor assistência médica); e asilo-refúgio (confundido como um lugar onde o idoso pode ter um espaço próprio, resguardando seu poder de decisão).

2 Os dados foram colhidos por meio de três formulários: a) Dados institucionais; b) Dados sociais dos idosos; c) Saúde geral. Esses formulários foram preenchidos em entrevista com os administradores, cuidadores e por meio das fichas individuais dos idosos.

3 Não houve possibilidade de acesso aos prontuários médicos dos idosos em razão de serem atendidos em diversas unidades de saúde. As informações acerca das patologias foram colhidas a partir da medicação de uso contínuo administrada aos internos e de entrevistas com os cuidadores.

4 A fim de evitar comparações entre as instituições, passa-se a apresentar os dados considerando a totalidade dos idosos $(\mathrm{n}=116)$.

5 Os dados apresentados foram aferidos com base nas seguintes fontes: prontuários dos idosos existentes nas IRLPIs pesquisadas; entrevistas com os cuidadores responsáveis pelo serviço de saúde e entrevista com alguns dos idosos.

6 Tal número foi variável no decorrer do programa, considerando que alguns idosos participam de forma esporádica, outros faleceram e outros foram integrados.
7 Os alunos do curso de Psicologia pertencem à Universidade de Marília. 